\title{
Reconhecimento e conduta de cirurgiões-dentistas diante de maus- tratos em crianças e adolescentes
}

\author{
Knowledge and attitudes of dentists regarding child and adolescent abuse \\ Reconocimiento y conducta de dentistas ante maltrato en niños y adolescentes
}

\author{
Adrianne Calixto Freire de Paula ${ }^{1 *}$, Bianca Oliveira de Carvalho ${ }^{2}$, Danielle Tayrine Celestino Fróes ${ }^{2}$, \\ Gisele Santos Ferreira ${ }^{2}$, Rodrigo Alves Pinto ${ }^{2}$, Thalita Thyrza de Almeida Santa-Rosa ${ }^{3}$ \\ Como citar esse artigo. de Paula, \\ ACF; de Carvalho, BO; Fróes, DTC;

\section{Resumo} Ferreira, GSF; Pinto, RA; Santa-Rosa, TTA. Reconhecimento e conduta de cirurgiões-dentistas diante de maustratos em crianças e adolescentes. Revista Pró-UniverSUS. 2019 Jan./Jun.; 10 (1): $137-144$

Este estudo objetivou identificar tipos de maus-tratos mais prevalentes incorridos contra crianças e adolescentes, características indicativas de maus-tratos observadas durante atendimento odontológico, além de descrever a conduta do cirurgião-dentista frente a esses casos. Os trabalhos científicos foram selecionados a partir da Biblioteca Virtual de Saúde; 24 artigos atenderam aos critérios pré-definidos. Um manual sobre notificação de maus-tratos infantis do Ministério da Saúde Brasileiro, e texto da Lei 8.069 também foram revisados. Observou-se que maus-tratos contra crianças e adolescentes comumente se manifestam em negligência, abusos físicos, sexuais e psicológicos. Ademais, constatou-se que o cirurgião-dentista pode exercer importante papel na identificação de maus-tratos infanto-juvenis, já que a maioria das lesões físicas oriundas de maus-tratos envolvem cabeça, pescoço e boca. Com base no Estatuto da Criança e Adolescente, o cirurgião-dentista é obrigado a identificar e notificar casos suspeitos de maus-tratos, encaminhando-os para o Conselho Tutelar. No entanto, notou-se inabilidade de cirurgiõesdentistas com relação ao diagnóstico e notificação. A subnotificação de casos de maus-tratos dificulta o dimensionamento epidemiológico do problema e consequente desenvolvimento de programas e ações para seu enfrentamento. Acredita-se que inserir o assunto "maus-tratos infanto-juvenis" nos projetos pedagógicos dos cursos, bem como divulgar práticas de notificação e conduta, contribuirão para erradicação desses maus-tratos.

Palavras-chave: Abuso infantil, Adolescentes; Cirurgião-dentista.

\begin{abstract}
This study aimed to identify: more prevalent types of maltreatment perpetrated against children and adolescents; suggestive features of maltreatment observed during dental treatment and to describe the attitudes of dentists towards these cases. The scientific works was selected from Virtual Health Library; 24 articles met the criteria. A manual about children maltreatment published by Brazilian Health Ministry and the text of the law 8.069 were also revised. It was observed that maltreatments against children and adolescents are expressed on neglect, physical, sexual and psychological abuse. Besides, it was found that dentist can play an important role in identifying maltreatment against children and adolescents, once many physical lesions coming from maltreatment involves head, neck and mouth. According to Adolescent and Child Statute, dentists are obligated to identify and notify suspected cases of maltreatment, referring them to Tutelary Council. However, dentists show inability in relation to diagnosis and notification of maltreatment cases. Underreporting maltreatment cases difficult epidemiological sizing of the problem and hence the development of programs and actions to face it. The authors believe that inserting the subject "children and adolescents maltreatment" on the courses pedagogical projects, besides propagating training on notification and attitudes can contribute to eradicate maltreatment.
\end{abstract}

Keywords: Child abuse, Adolescents, Dentist.

\section{Resumen}

El objetivo de este estudio fue identificar: los tipos más frecuentes de maltrato perpetrado contra niños y adolescentes; las características sugerentes de maltrato observadas durante el tratamiento dental y describir las actitudes de los dentistas hacia estos casos. Los trabajos científicos fueron seleccionados de la Biblioteca Virtual en Salud; 24 artículos cumplieron los criterios. También se revisó un manual sobre maltrato infantil publicado por el Ministerio de Salud de Brasil y el texto de la ley 8.069. Se observó que los malos tratos contra niños y adolescentes se expresan sobre negligencia, abuso físico, sexual y psicológico. Además, se encontró que el dentista puede desempeñar un papel importante en la identificación del maltrato contra niños y adolescentes, una vez que muchas lesiones físicas provenientes del maltrato involucran cabeza, cuello y boca. De acuerdo con el Estatuto de los adolescentes y los niños, los dentistas están obligados a identificar y notificar los casos sospechosos de maltrato, remitiéndolos al Consejo Tutelar. Sin embargo, los dentistas muestran incapacidad en relación con el diagnóstico y la notificación de casos de maltrato. La notificación insuficiente de casos de maltrato dificulta el dimensionamiento epidemiológico del problema y, por ende, el desarrollo de programas y acciones para enfrentarlo. Los autores creen que insertar el tema "maltrato de niños y adolescentes" en los proyectos pedagógicos de los cursos, además de difundir la capacitación sobre notificación y actitudes, puede contribuir a erradicar el maltrato.

Palabras clave: Abuso infantil, Adolescentes, Dentista.

\section{Afiliação dos autores:}

1Cirurgiã-dentista. Doutora em Odontologia em Saúde Coletiva pela Faculdade de Odontologia UFMG. Docente do Departamento de Odontologia da Unimontes. MG, Brasil. Email: thalitasantarosa@yahoo.com.br ORCID: https://orcid.org/0000-0002-2289-993X

2 Cirurgiã-Dentista. Doutora em Endodontia pela Faculdade de Odontologia de Ribeirão Preto USP. Professora do Departamento de Odontologia da Unimontes. MG, Brasil. Email: adriannepaula@bol.com.br ORCID: https://orcid.org/0000-0002-6841-8556

3Acadêmico em Odontologia da Unimontes. MG, Brasil. Email: biancaoliveiradecarvalho@hotmail.com ORCID: https://orcid.org/0000-0002-9698-2357

4Acadêmico em Odontologia da Unimontes. MG, Brasil. Brasil. Email: dani-tayrine@hotmail.com ORCID: https://orcid.org/0000-0003-4239-934X

5Acadêmico em Odontologia da Unimontes. MG, Brasil. Brasil. Email: giselef_dossantos@hotmail.com ORCID: https://orcid.org/0000-0002-8429-8187

6Acadêmico em Odontologia da Unimontes. MG, Brasil. Email: rodrigo.alves1789@gmail.com ORCID: https://orcid.org/0000-0002-2778-9991

* Email de correspondencia: thalitasantarosa@yahoo.com.br

Recebido em: 11/03/18. Aceito em: 02/05/19. 


\section{Introdução}

Os maus-tratos contra crianças e adolescentes são conOs maus-tratos contra crianças e adolescentes são considerados problemas sociais e de saúde pública. Esses maus-tratos podem apresentar-se como abuso físico, sexual, psicológico ou negligência e influenciam diretamente o desenvolvimento cognitivo, social e afetivo das crianças e adolescentes ${ }^{1}$. Frequentemente, os termos maus-tratos contra crianças e adolescentes são usados como sinônimos da expressão abuso infantojuvenil².

No Brasil, os maus-tratos foram considerados, em 2013, a primeira causa mais prevalente de morte para a faixa etária de cinco a dezenove anos e a segunda para a faixa etária de um a quatro anos de idade 3 . Além disso, em 2017, o país foi apontado como a quinta nação com as maiores taxas de violência contra crianças e adolescentes, de acordo com um relatório do Fundo das Nações Unidas para a Infância ${ }^{4}$.

O tipo mais frequente de mau trato infantojuvenil é a violência doméstica, que costuma ter caráter prolongado uma vez que o protetor da criança tende a acobertar o ato de violência. Entende-se como violência doméstica toda conduta que prejudica a integridade física, psicológica, emocional, o bem-estar ou a liberdade e o direito ao pleno desenvolvimento de qualquer pessoa que habite o mesmo agregado familiar, em especial crianças e adolescentes ${ }^{3}$. Deve ser salientado ainda que, de acordo com o Estatuto da Criança e do Adolescente (ECA), criança é a pessoa com até doze anos de idade incompletos, e adolescentes são aqueles que possuem entre doze e dezoito anos de idade 5 .

O profissional de saúde, de um modo geral, tem papel de destaque no reconhecimento e no manejo de situações de maus-tratos dirigidos contra a população infanto-juvenil. Como a maioria das lesões físicas (por exemplo, hematomas, queimaduras, lacerações, cortes e contusões) oriundas de maus-tratos envolvem as regiões de cabeça, pescoço e boca, acredita-se que o cirurgiãodentista possua grande importância na identificação e notificação dessas situações3. Ressalta-se que os profissionais de saúde têm a obrigação legal, ética e moral de realizar a notificação dos casos suspeitos de maus-tratos, com base na Constituição Federal do Brasil e no ECA $^{5}$. Além disso, a notificação de maus-tratos contribui para o dimensionamento epidemiológico do problema, permitindo o desenvolvimento de programas e ações mais condizentes com a realidade ${ }^{3}$.

É reconhecido, todavia, que alguns cirurgiõesdentistas não notificam casos suspeitos de maus-tratos por medo de perder pacientes, falta de confiança no serviço de proteção à criança e aos adolescentes, medo de lidar com os pais, incerteza do diagnóstico e desconhecimento da verdadeira responsabilidade em denunciar esses $\operatorname{casos}^{1}$. Nesse aspecto, há que se considerar a necessidade de familiarizar estudantes de Odontologiae cirurgiões-dentistas comos procedimentos de diagnóstico e notificação de maus-tratos infantojuvenis, com base na legislação relacionada a esse assunto.

Esta revisão de literatura objetiva identificar os tipos de maus-tratos mais prevalentes incorridos contra crianças e adolescentes, as características indicativas de maus-tratos observadas durante o atendimento odontológico, além de descrever a conduta do cirurgiãodentista frente a esses casos.

\section{Materiais e Métodos}

Os trabalhos científicos foram selecionados a partir da base de dados Biblioteca Virtual de Saúde (BVS), utilizando os descritores: "abuso infantil"; "cirurgião-dentista"; "child abuse"; "dentist".

A escolha dos trabalhos ocorreu em três etapas. $\mathrm{Na}$ primeira, a seleção foi feita de acordo com os critérios: (1) pertencer a língua portuguesa ou a língua inglesa; (2) ter publicação feita entre 2008 e janeiro de 2018; e (3) estar disponível na íntegra nas bases pesquisadas. Quarenta e nove trabalhos atenderam aos critérios supracitados.

$\mathrm{Na}$ segunda etapa, os resumos dos quarenta e nove trabalhos foram lidos e as seguintes publicações foram excluídas: (1) disponíveis apenas em versão paga; (2) em que o objetivo se distanciava do propósito deste trabalho; e (3) do tipo carta ao editor, editorial, notícia, entrevista, relato de caso e pesquisa ação. Após essa etapa, vinte e quatro estudos foram mantidos.

$\mathrm{Na}$ terceira etapa, os vinte e quatro estudos selecionados anteriormente foram lidos na íntegra e avaliados com relação a adequação com o tema proposto nesta revisão de literatura. Todos os trabalhos foram considerados adequados, passando a compor o subsequente trabalho. Desses artigos, 83,3\% apresentaram delineamento transversal e 16,7\% constituíram revisões de literatura. Verificou-se que a maioria dos artigos selecionados têm origem no Brasil $(54,1 \%)$, seguido pelo Reino Unido, (12,5\%), Croácia $(8,3 \%)$, Índia $(8,3 \%)$, Arábia Saudita $(4,2 \%)$, Dinamarca $(4,2 \%)$, Jordânia $(4,2 \%)$ e Portugal $(4,2 \%)$. Informações sobre o país de origem e tipo de estudo são apresentadas na Figura 1. A maior parte dos trabalhos foi publicada em $2008(20,8 \%)$ e $2015(20,8 \%)$. Além disso, houve publicações em $2013(16,7 \%), 2016(12,6 \%), 2010$ (8,3\%), $2011(8,3 \%), 2012(8,3 \%)$ e $2018(4,2 \%)$.

Além dos artigos encontrados na BVS, foram localizados um manual sobre notificação de maus-tratos infantis, publicado pelo Ministério da Saúde, em 2001, e o texto da lei n ${ }^{\circ} 8.069$, de 13 de julho de 1990, que dispõe sobre o ECA.5,6 Em vista de seu caráter regulamentar, 
Figura1. Tipos de estudo e países de publicação dos trabalhos usados na revisão de literatura

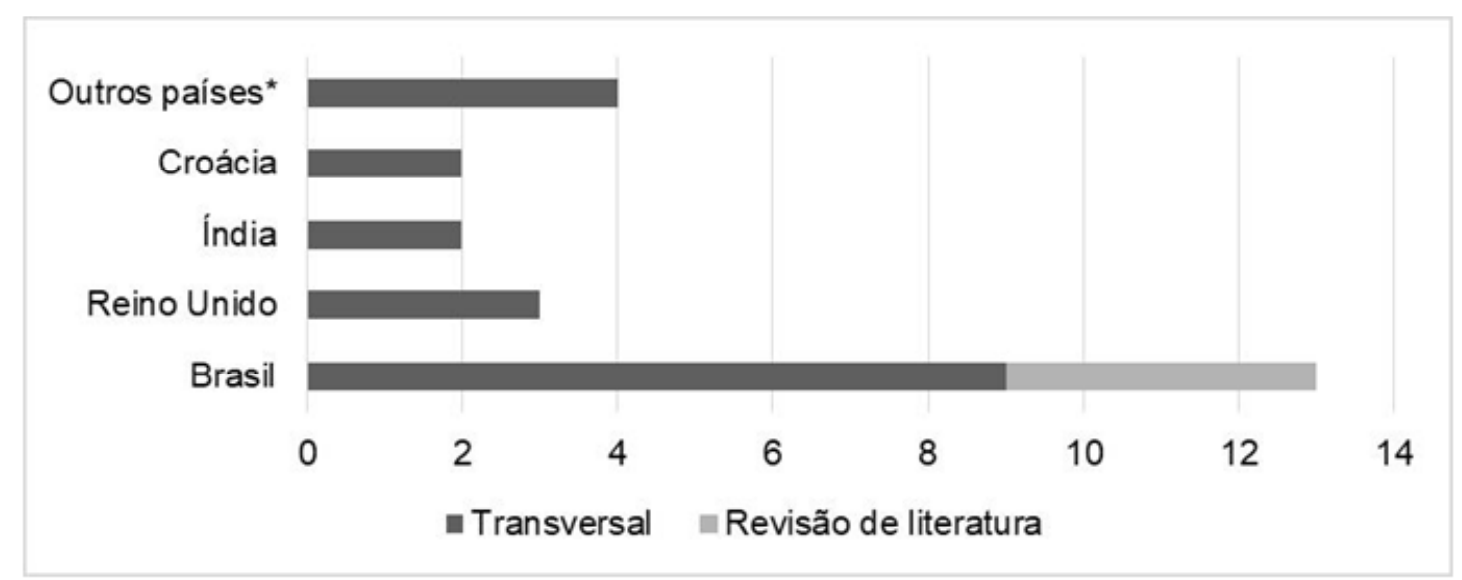

*Arábia Saudita: 1 trabalho; Dinamarca: 1 trabalho; Jordânia: 1 trabalho; Portugal: 1 trabalho

considerou-se pertinente inserir informações do manual e da lei n ${ }^{\circ} 8.069$ nesta revisão de literatura.

\section{Resultados e Discussão}

\section{Maus-tratos contra crianças e adolescentes: tipos mais prevalentes e características sugestivas observadas durante $o$ atendimento odontológico}

Ao fazer diagnóstico de lesões bucais oriundas de maus-tratos, o cirurgião-dentista deve considerar que essas lesões não têm seu impacto restrito apenas a cavidade bucal. Além disso, quando a violência é tratada no âmbito da saúde, o cirurgião-dentista deve reconhecer que uma abordagem diferenciada se torna necessária, incluindo conhecimentos já consolidados nas ciências sociais e diluindo a clássica divisão entre clínica e saúde pública, já que técnicas terapêuticas convencionais, bem como ações de prevenção e de promoção de saúde são demandadas ${ }^{6}$.

Os maus-tratos contra crianças e adolescentes têm uma forte carga cultural. Por muito tempo, acreditou-se que para educar crianças e adolescentes seria necessário puni-los quando errassem ou se insubordinassem. Os maus-tratos que ocorrem dentro das famílias são potencializadores da violência social em geral e, comumente, não constituem um ato isolado. As crianças e adolescentes vítimas de maus-tratos tendem a repetilos com outras crianças e adolescentes com as quais se relacionam socialmente e/ou quando se tornarem adultos, especialmente com seus próprios filhos ${ }^{6,7}$.

É sabido que maus-tratos infanto-juvenis podem ser apresentados ao cirurgião-dentista através de diferentes maneiras: (1) por meio de uma alegação direta da criança, adolescente, responsável ou outra pessoa; (2) através de sinais e sintomas que são sugestivos de maustratos; (3) através de observações do comportamento da criança e do adolescente ou da interação entre eles e seus pais ${ }^{2}$.

Dito isso, alguns indicadores físicos sugestivos de maus-tratos podem ser observados na rotina odontológica, dos quais: laceração do freio labial, traumas dentários recorrentes, traumas na cabeça, hematomas, queimaduras, marcas de mordida e lesões cariosas de grau avançado em muitos dentes ${ }^{7-12}$. Além disso, também é importante considerar que os maustratos podem se manifestar de muitas formas; os tipos mais frequentes de maus-tratos são mencionados a seguir:

Negligência e abandono. Omissões dos pais ou de outros responsáveis, inclusive institucionais, quando deixam de prover as necessidades básicas para o desenvolvimento físico, emocional e social das crianças e adolescentes. $\mathrm{O}$ abandono é considerado uma forma de negligência extrem $2,6,9,11,13,14$.

AA negligência relativa à saúde bucal da criança e do adolescente é o tipo de mau trato mais frequentemente identificado no âmbito odontológico. Ocorrência de cáries dentárias, doença periodontal e outras condições desfavoráveis para a saúde da boca podem causar dor, infecção, bem como perda de função e consequente impacto na qualidade de vida da criança e do adolescente. É necessário salientar que muitas famílias encontram problemas para acessar serviços de assistência odontológica, sendo assim o cirurgiãodentista deve avaliar se os serviços de cuidado bucal estão acessíveis para a criança e sua família antes de determinar a ocorrência de negligência ${ }^{1,2,9,15}$.

Abusos físicos. Atos violentos com uso da força física de forma intencional, não acidental, praticada por pais, responsáveis, familiares ou pessoas próximas da criança ou do adolescente, com o objetivo de ferir a vítima, deixando ou não marcas evidentes em seu corpo $^{2,6,11,13,14}$. Lesões geradas por maus-tratos em crianças e adolescentes ocorrem frequentemente nas regiões da cabeça, face e pescoço, pois essas áreas são expostas e de fácil acesso ${ }^{7-12}$.

Abuso sexual. Ato ou jogo sexual envolvendo crianças ou adolescentes e cujo agressor está em estágio 
de desenvolvimento psicossocial mais adiantado do que a criança ou adolescente. Apresenta-se sobre a forma de práticas eróticas e sexuais impostas pela violência física, ameaças ou indução da vontade, e pode variar desde atos em que não se produz o ato sexual (voyeurismo, exibicionismo, produção de fotos), até diferentes tipos de ações que incluem contato sexual com ou sem penetração. Engloba ainda a situação de exploração sexual visando lucros, como é o caso da prostituição e da pornografia ${ }^{6,11,13,16,17}$. A presença de doenças venéreas em crianças e adolescentes pode ser indicativa de abuso sexual ${ }^{1}$.

Abuso psicológico. Toda forma de rejeição, depreciação, discriminação, desrespeito, cobranças exageradas, punições humilhantes e utilização da criança ou do adolescente para atender às necessidades psíquicas do adulto. Pela falta de materialidade do ato, que atinge sobretudo o campo emocional da vítima, e pela falta de evidências imediatas de maustratos, esse tipo de violência é um dos mais difíceis de ser identificado. Medo, raiva, ansiedade, depressão, angústia, baixa autoestima, desconfiança, isolamento, além de mentiras frequentes e redução no rendimento escolar são características que podem indicar abuso psicológico ${ }^{6,17}$.

Salienta-se, no entanto, que para levantar suspeita demaus-tratos, os sinais indicados anteriormente não devem ser analisados de forma isolada. Sendo assim, deve-se considerar que crianças e adolescentes normalmente só falam sobre os episódios de maus-tratos muito tempo depois de eles terem ocorrido; portanto, quando uma criança ou um adolescente declara que foi vítima de maus-tratos, a acusação deve ser imediatamente investigada. Em muitos casos, o agente dos maus-tratos é alguém que a criança ou adolescente conhece bem. No entanto, não se deve confrontar diretamente o agente suspeito dos maus-tratos; a princípio, deve-se investigar a situação cautelosamente e expor os sinais observados que são sugestivos de maus-tratos, sem indicar alguém como culpado pelas lesões encontradas ${ }^{8,10,12}$.

Deve-se pontuar que maus-tratos contra crianças e adolescentes ocorrem tanto em grupos socioeconomicamente desfavorecidos quanto em grupos socioeconomicamente favorecidos ${ }^{12,18}$. Matos e colaboradores (2013), todavia, encontraram que algumas características sociodemográficas podem se tornar agravantes no que diz respeito a prevalência de maus-tratos, como a baixa renda, famílias jovens e histórico anterior de maus-tratos na família ${ }^{19}$.

Além dos tipos mais prevalentes e características sugestivas de maus-tratos observadas durante 0 atendimento odontológico, torna-se importante conhecer as obrigações legais do cirurgião-dentista diante de casos suspeitos de maus-tratos contra crianças e adolescentes.

\section{Obrigações legais}

Em 1988, a Constituição Brasileira reconheceu, no seu artigo 277, que crianças e adolescentes são portadores de direitos especiais, modificando a legislação anterior que os considerava propriedade dos seus pais. Mais tarde, em 1990, o ECA foi regulamentado, exigindo que crianças e adolescentes fossem criados de forma saudável e respeitosa, impondo prioridade por parte da família, da sociedade e do Estado às necessidades deles e determinando que eles precisam de ser protegidos em decorrência do seu estado de desenvolvimento físico, moral e psicológico ${ }^{5,6}$.

O ECA também possui leis específicas sobre o papel dos profissionais de saúde com relação aos maus-tratos contra crianças e adolescentes. Seu artigo 11 incumbe os profissionais de saúde de identificar e de notificar situações de maus-tratos. O artigo 245 define como infração administrativa, sujeita a multa de três a vinte salários vigentes, a não referência e a não comunicação à autoridade competente, pelo profissional de saúde ou responsável pelo estabelecimento de atenção à saúde, dos casos de maus-tratos à criança e ao adolescente sobre os quais tenha conhecimento ${ }^{5,6}$.

A Portaria n ${ }^{\circ}$ 1968/2001 do Ministério da Saúde torna obrigatória, para todas as instituições de saúde pública e/ou conveniadas ao Sistema Único de Saúde, o preenchimento da "Ficha de Notificação de Suspeita ou Confirmação de Maus-tratos contra Crianças e Adolescentes" (Figura 2) e seu encaminhamento aos órgãos competentes. O artigo 13 do ECA define que os encaminhamentos de casos de maus-tratos contra crianças e adolescentes devem ser feitos ao Conselho Tutelar. Nos municípios que ainda não possuem um Conselho Tutelar, os casos de maus-tratos e de outras violações de direitos da criança e do adolescente costumam ser encaminhados para as Varas da Infância e Juventude, ou para outra Vara, no caso da não existência de uma Vara especializada, ou ainda para a Promotoria Pública. Salienta-se que a notificação de casos de maustratos contra crianças e adolescentes não constitui uma denúncia policial ${ }^{2,5,6,9,20}$. A Figura 3 apresenta um resumo sobre o processo de notificação de casos de maus-tratos contra crianças e adolescentes.

O Código de Ética Odontológico (CEO) não determina, especificamente, que o cirurgião-dentista deve notificar situações suspeitas de maus-tratos contra crianças e adolescentes. Todavia, esse dever é corroborado quando o CEO impõe que o cirurgiãodentista deve colaborar com a justiça nos casos previstos em lei ${ }^{9}$.

Apesar de o ECA determinar como obrigatória a notificação de maus-tratos contra crianças e adolescentes, acredita-se que essa obrigação é escassamente cumprida. Historicamente, observa-se que alguns profissionais de saúde se limitaram a cuidar 


\section{FICHA DE NOTIFICAÇÃO DE SUSPEITA OU CONFIRMAÇÃO DE MAUS- TRATOS CONTRA CRIANÇAS E ADOLESCENTES}

(Considera-se criança a pessoa até 12 anos de idade incompletos e adolescente aquela entre 12 e 18 anos de idade -Lei n. ${ }^{\circ} 8.069$, de 13/7/90 - Estatuto da Criança e do adolescente)

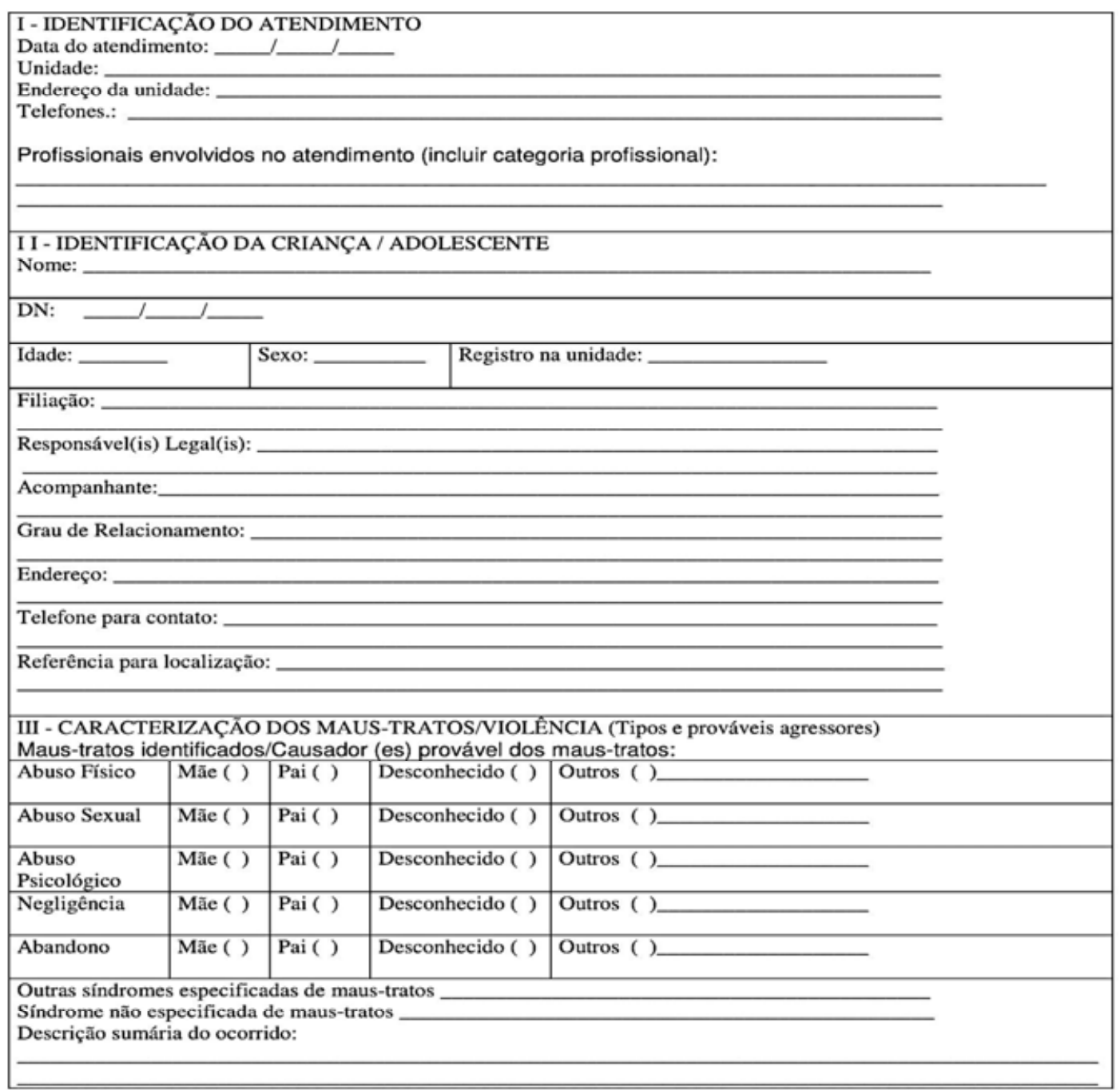

Figura2. Ficha de Notificação de Suspeita ou Confirmação de Maus-tratos contra Crianças e Adolescentes.

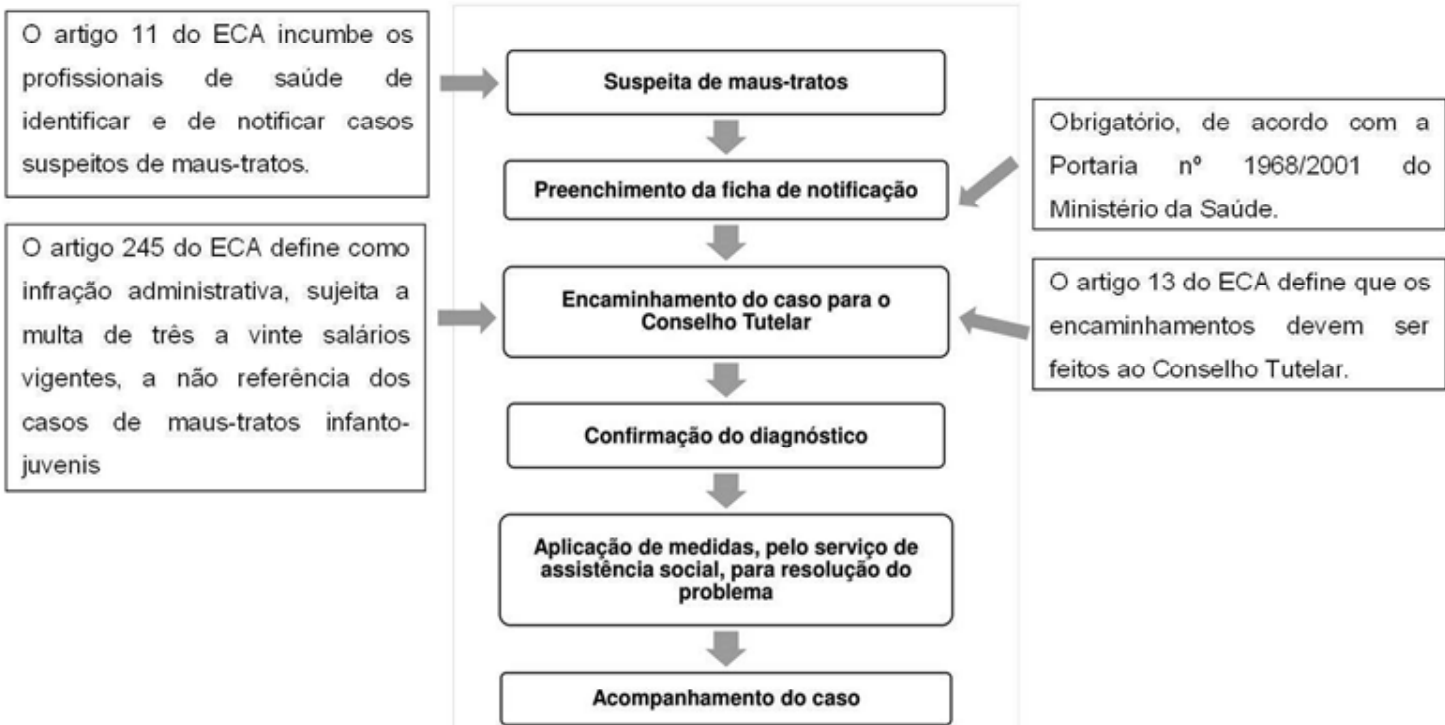

Figura3. Organograma sobre o processo de notificação de casos de maus-tratos contra crianças e adolescentes. 
apenas dos problemas físicos oriundos de maus-tratos, como lesões e traumas. É possível que profissionais de saúde não tenham oportunidades suficientes para estudar sobre violência e maus-tratos na faculdade, situação que deve ser pesquisada. Talvez, por isso, eles encontrem dificuldades tanto para fazer o diagnóstico como para tomar a conduta adequada quando se deparar com situações de maus-tratos contra crianças e adolescentes. Mesmo os profissionais sensíveis ao impacto dos maustratos sobre o crescimento e o desenvolvimento de crianças e adolescentes podem realizar um atendimento apressado ou pouco aprofundado. Isso se deve, de um lado, à enorme demanda que os absorvem e, de outro, à pouca experiência com esse tipo de problema. Por isso, é muito importante que os gestores de saúde criem estratégias que facilitem a implantação de boas práticas de assistência e de notificação de maus-tratos ${ }^{6,20}$.

\section{Conduta dos cirurgiões-dentistas frente aos maus-tratos contra crianças e adolescentes}

A inabilidade de cirurgiões-dentistas com relação ao diagnóstico e à notificação de maus-tratos contra crianças e adolescentes é um problema internacional. Os resultados de estudos conduzidos fora do Brasil mostraram que pequena porcentagem de cirurgiõesdentistas diagnostica e/ou notifica casos suspeitos de maus-tratos contra crianças e adolescentes. Nos diferentes estudos estrangeiros revisados, observou-se que entre $10-38 \%$ dos cirurgiões-dentistas já haviam suspeitado de casos de maus-tratos infanto-juvenis, todavia apenas $3-12 \%$ desses profissionais notificaram esses casos para as autoridades competentes ${ }^{8,10,18,23,24,25}$.

Os achados de estudos conduzidos no Brasil também demonstraram que cirurgiões-dentistas brasileiros apresentam dificuldade de manejar casos suspeitos de maus-tratos infanto-juvenis. Azevedo e colaboradores (2012), em um estudo realizado no Rio Grande do Sul, observaram que $14,3 \%$ dos cirurgiõesdentistas avaliados declararam já ter diagnosticado um caso de mau trato infanto-juvenil, no entanto, $76 \%$ deles admitiram não ter notificado os casos para o Conselho Tutelar ${ }^{2}$. Nessa mesma perspectiva, em um estudo realizado no Ceará, observaram que 28,3\% dos cirurgiões-dentistas avaliados afirmaram que haviam identificado situações de maus-tratos na sua prática profissional. Desses, $83,1 \%$ não notificaram as ocorrências ${ }^{20}$. Outros estudos brasileiros demonstraram que quantidade razoável de cirurgiões-dentistas reconhece sua obrigação legal de notificar casos de maus-tratos infanto-juvenis (43\%) e sabe que esses casos devem ser notificados para o Conselho Tutelar $(42-63 \%)^{1,16,26}$. Em vista disso, acredita-se que possa haver significativa diferença entre o conhecimento da obrigação de se notificar casos suspeitos de maus-tratos infanto-juvenis e a conduta, isto é, a aplicação prática desse conhecimento.
Há concordância entre estudos brasileiros e estrangeiros sobre as razões apresentadas pelos cirurgiões-dentistas com relação a resistência em notificar casos suspeitos de maus-tratos infanto-juvenis. As razões apresentadas pelos cirurgiões-dentistas incluem: medo de impacto no trabalho, medo de serem processados, medo de violência da família contra eles, medo de a notificação motivar mais episódios de maus-tratos contra a criança ou ao adolescente, falta de conhecimento acerca dos procedimentos para notificação, falta de certeza com relação ao diagnóstico e descrença nos serviços de apoio social para os quais os casos são encaminhados $1,2,8,10,12,13,15,18,19,23,24,25$.

Um estudo27 em 1998, também observou que os profissionais de saúde apresentavam resistência para notificar situações de maus-tratos contra crianças e adolescentes, apoiando-se em motivos semelhantes aos citados anteriormente. Isso pode indicar que o tema "diagnóstico e notificação de maus-tratos contra crianças e adolescentes" não tem recebido atenção adequada. Também aponta que alguns receios apresentados pelos profissionais de saúde com relação a notificação de maus-tratos precisam de ser descontruídos, com base na legislação vigente e no compromisso ético e moral desses profissionais de oferecer o cuidado a vítimas de maustratos. Salienta-se que, no Brasil, a legislação garante imunidade criminal ou civil quando profissionais fazem um relato de boa-fé da situação sugestiva de maus-tratos encontrada ${ }^{2}$.

Alguns estudos relataram associações interessantes entre o perfil do cirurgião-dentista, a frequência de identificação e o conhecimento sobre sinais e sintomas presentes em casos de maus-tratos infanto-juvenis. Observaram que cirurgiões-dentistas que trabalhavam em serviços públicos suspeitaram com mais frequência de casos de maus-tratos infantojuvenis $^{23}$. Nessa mesma perspectiva, Outro estudo ${ }^{10}$ observou que trabalhar em serviços públicos de saúde aumenta as chances de se conhecer melhor os sinais e sintomas de maus-tratos infantis e a conduta que deve ser tomada frente a esses $\operatorname{casos}^{10}$. A literatura aponta ainda que, uma proporção significativamente maior de cirurgiões-dentistas trabalhando em universidades já havia identificado casos suspeitos de maus-tratos contra crianças2. É possível que esses achados se justifiquem devido ao fato de que cirurgiões-dentistas que trabalham em universidades e serviços públicos veem um número maior de pacientes. Também se acredita que cirurgiões-dentistas que trabalham em universidades são devidamente treinados para lidar com situações suspeitas de maus-tratos contra crianças e adolescentes.

Diante desses achados, acredita-se que experiência e educação são determinantes para o conhecimento sobre o diagnóstico e a notificação de casos suspeitos de maus-tratos infanto-juvenis. Em acordo com essa 
hipótese, um estudo ${ }^{11}$, ao avaliarem odontopediatras, profissionais especializados e capacitados para lidar com situações relativas à saúde bucal de crianças, observaram que $93,5 \%$ e $77 \%$ desses profissionais, respectivamente, declararam saber notificar e agir diante de casos suspeitos de maus-tratos contra crianças.

Por fim, também há que se considerar a necessidade de conscientizar os cirurgiões-dentistas acerca de seu papel na prevenção e notificação dos casos de maus-tratos, uma vez que pode haver diferença entre conhecer obrigações e agir diante desses casos.

\section{Considerações Finais}

Conclui-se que os maus-tratos contra crianças e adolescentes têm uma forte carga cultural, comumente se manifestando em forma de negligência, abusos físicos, sexuais e psicológicos. O cirurgião-dentista pode exercer importante papel na identificação de casos de maus-tratos infanto-juvenis, já que a maioria das lesões físicas oriundas de maus-tratos envolvem as regiões da cabeça, pescoço e boca.

Alguns indicadores objetivos que estão relacionados à ocorrência de maus-tratos podem ser observados na rotina odontológica, dos quais: laceração do freio labial, traumas dentários recorrentes, traumas na cabeça, hematomas, queimaduras, marcas de mordida e lesões cariosas de grau avançado em muitos dentes. Indicadores subjetivos que sugerem ocorrência de maus-tratos são: medo, raiva, ansiedade, depressão, angústia, baixa autoestima, desconfiança, isolamento, além de mentiras frequentes e redução no rendimento escolar. Para levantar suspeita de maus-tratos, todavia, esses indicadores não devem ser analisados de forma isolada.

Combase no Estatuto da Criança e doAdolescente, o cirurgião-dentista é obrigado a identificar e a notificar casos suspeitos de maus-tratos, encaminhando-os para o Conselho Tutelar. No entanto, a inabilidade de cirurgiões-dentistas com relação ao diagnóstico e à notificação de maus-tratos contra crianças e adolescentes é um problema internacional.

Há que se considerar, portanto, a necessidade de familiarizar estudantes de Odontologia e cirurgiõesdentistas com os procedimentos de diagnóstico e notificação de maus-tratos infanto-juvenis, com base na legislação relacionada a esse assunto. Acredita-se que inserir tal temática nos projetos pedagógicos dos cursos, bem como divulgar práticas de notificação e conduta diante de casos de maus-tratos, sejam um caminho para contribuir com a erradicação dos maus-tratos.

\section{Agradecimentos}

À Fundação de Amparo à Pesquisa do Estado de Minas Gerais - FAPEMIG e Universidade Estadual de
Montes Claros pela concessão de bolsas de Iniciação Científica.

\section{Referências}

1. Granville-Garcia AF, Silva MJF, Menezes VA. Maus-tratos infantis: percepção e responsabilidade do cirurgião do cirurgião-dentista. Rev Odonto Ciênc. 2008; 23 (1): 35-39.

2. Azevedo MS et al. Child maltreatment: a survey of dentists in Southern Brazil. Brazilian Oral Research. 2012 jan; 26 (1): 1-7.

3. Ayres KCM. Violência doméstica, perfil da vítima e o papel do dentista: Uma revisão crítica da literatura. Monografia apresentada à Faculdade de Odontologia de Piracicaba da Universidade Estadual de Campinas, Piracicaba. 2013. 22 páginas.

4. United Nations Children's Fund. A Familiar Face: Violence in the lives of children and adolescents. UNICEF: New York, 2017.

5. Brasil. Lei n. 8069 de 13 de julho de 1990. Estatuto da Criança e do Adolescente. Brasília: Presidência da República, 1990.

6. Brasil. Ministério da Saúde. Secretaria de Assistência à Saúde Notificação de maus-tratos contra crianças e adolescentes pelos profissionais de saúde: um passo a mais na cidadania em saúde. Série A. Normas e Manuais Técnicos. Brasília: Ministério da Saúde, 2001.

7. Herrera LM, Serra MC, Fernandes CMS. Violence against children and adolescents: the importance of knowledge of the spectrum of bruise colors in its diagnosis. RSBO. 2013 out-dez; 10 (4): 378-385.

8. Al-Habsi SA. et al. A survey of attitudes, knowledge and practice of dentists in london towards child protection: are children receiving dental treatment at the Eastman Dental Hospital likely to be on the child protection register? British Dental Journal. 2008 nov; 01-05.

9. Massoni ACLT et al. Aspectos orofaciais dos maus-tratos infantis e da negligência odontológica. Ciência e Saúde Coletiva. 2010 mar; 15 (2): 403-410.

10. Mogaddam, M. et al. Knowledge, attitudes, and behaviors of dentists regarding child physical abuse in Jeddah, Saudi Arabia. Science Direct. 2016 abril; 16: 43-56.

11. Santos CAO et al. Violência contra crianças e adolescentes: conhecimentos dos odontopediatras da capital paraibana - estudo piloto. Rev Odontol Univ. 2016 set-dez; 28(3): 223-229.

12. Anshula D et al. Knowledge and attitude in regards to physical child abuse amongst medical and dental residents of central gujarat: a crosssectional survey. Journal of Indian Society of Pedodontics and Preventive Dentistry. 2015 jul-set; 33 (3): 177-182.

13. Kaur $\mathrm{H}$ et al. Child abuse: cross-sectional survey of general dentists. J Oral Biol Craniofac Res. 2015 mar-ag; 6(2): 118-123.

14. Sales-Peres A et al. Odontologia e o desafio na identificação de maustratos. Odontol Clín-Científ. 2008 jul-set; 7 (3): 185-189.

15. Markovic $\mathrm{N}$ et al. Knowledge and attitudes regarding child abuse and neglect. Mater Sociomed, Bosnia and Herzegovina. $2015 \mathrm{dez} ; 27$ (6): 372375 .

16. Moura AR et al. Dentists and undergraduate dental students require more information relating to child abuse. Medicalexpress, São Paulo. 2015 mar-abr; 2 (2).

17. Alves MA et al. Importância do cirurgião-dentista no diagnóstico de abuso sexual infantil - revisão de literatura. Revista Brasileira de Odontologia Legal. 2016 jan; 92-99.

18. Harris CM, Welbury R, Cairns AM. The Scottish dental practitioner's role in managing child abuse and neglect. Br Dent J. 2013 mai; 214 (9): 378385 .

19. Matos FZ et al. Avaliação do conhecimento dos alunos de graduação em odontologia x cirurgião dentista no diagnóstico de maus-tratos a crianças. Rev Odontol Bras Central. 2013 jan. 
20. Moreira GAR et al. Instrumentação e conhecimento dos profissionais da Equipe Saúde da Família sobre a notificação de maus-tratos em crianças e adolescentes. Rev Paul Pediatr. 2013 jan; 31 (2): 223-230.

21. Harris JC et al. NHS dental professionals' evaluation of a child protection learning resource. British Dental Journal. 2011 jan; 210 (2): 7579.

22. Moreira GAR et al. Atuação do cirurgião-dentista na identificação de maus-tratos contra crianças e adolescentes na atenção primária. Saúde Debate. 2015 dez; 39: 257-267.

23. Uldum B et al. Danish dentists' and dental hygienists' knowledge of and experience with suspicion of child abuse or neglect. International Journal Of Paediatric Dentistry. 2010 ago; 20 (5): 361-365.

24. Sonbol $\mathrm{HN}$ et al. Knowledge, educational experiences and attitudes towards child abuse amongst Jordanian dentists. Eur J Dent Educ. 2012 fev; 16 (1): $158-165$

25. Cukovic-Bagic I et al. Croatian dentists' knowledge, experience, and attitudes in regard to child abuse and neglect. Int J Paediatr Dent. 2015 nov; 25 (6): 444-450

26. Fracon ET, Silva RHA, Bregagnolo C. Avaliação da conduta do cirurgião-dentista ante a violência doméstica contra crianças e adolescentes no município de Cravinhos (SP). RSBO. 2011 abr-jun; 8(2): 153-159.

27. Almeida EC. Violência doméstica: um desafio para a formação do pediatra. Dissertação de mestrado, Instituto de Medicina Social, Universidade do Estado do Rio de Janeiro. Rio de Janeiro. 1998 